\title{
Numerical Solution of a Kidney Model by Multiple Shooting
}

\author{
PETER LORY \\ Institut für Mathematik, Technische Universität München, \\ Arcisstrasse 21, D-8000 München 2, Federal Republic of Germany
}

Received 2 March 1979; revised 21 December 1979

\begin{abstract}
The mathematical modeling of the renal counterflow system involves a two-point boundary-value problem for a system of nonlinear differential equations. In this paper the multiple shooting technique is used for the numerical solution of this problem. This method ensures highly precise and reliable computations. Special consideration is given to the treatment of the border between outer and inner medulla for various widths of the outer zone.
\end{abstract}

\section{INTRODUCTION}

The mathematical models of the mechanism for urine concentration in the kidney contribute towards a better understanding of the renal function. The work presented in this paper deals with a six-tube model of the medullary counterflow system. The model includes provisions for all the tubes present in the renal medulla and was suggested by Stephenson, Tewarson, and Mejia [1]. The mathematical description involves a twopoint boundary-value problem for a system of nonlinear differential equations.

For the numerical solution of this problem the multiple shooting technique is used in this paper. This method is described in detail in Bulirsch, Stoer, and Deuflhard [2], Stoer and Bulirsch [3], and Keller [4]. It ensures highly precise and reliable computations. Special consideration is given to the treatment of the border between outer and inner medulla for various widths of the outer zone.

The paper is structured as follows: In Sec. 2 the mathematical model is outlined. Section 3 contains a description of the multiple shooting method for the solution of two-point boundary-value problems. In Sec. 4 the numerical results are presented. 


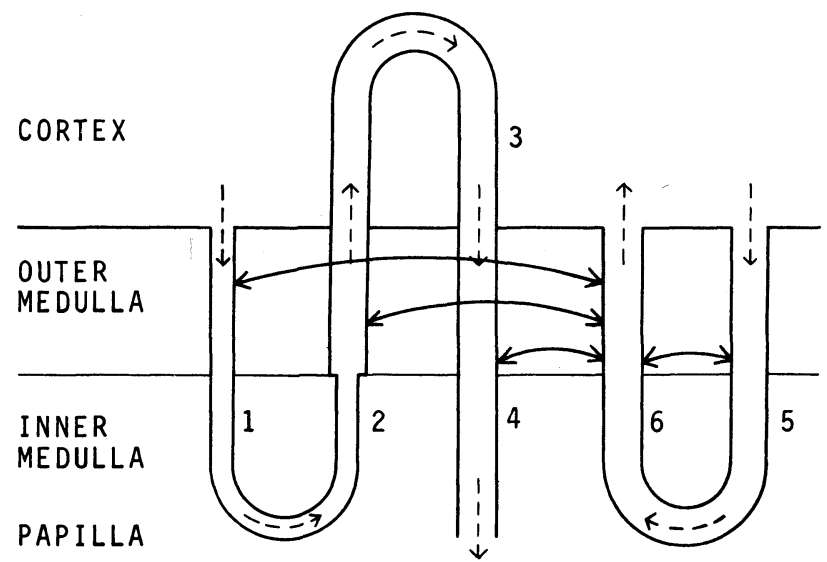

Fig. 1. The six-tube model of the medullary counterflow system with the axial flows (dashed arrows) and the transmural fluxes (solid arrows).

\section{THE MATHEMATICAL MODEL}

In the six-tube model of the medullary counterflow system (Fig. 1), fluid from the proximal tubule enters tube 1 (the descending Henle's limb-DHL). Then it flows through tube 2 (the ascending Henle's limbAHL), tube 3 (the distal nephron-DN), and tube 4 (the collecting duct$\mathrm{CD}$ ) in sequence to emerge from tube 4 as final urine. Blood enters tube 5 (the descending vasa recta-DVR) and leaves from tube 6 (the ascending vasa recta-AVR). Tube 3 exchanges solutes and water with the cortical interstitium - a bath of uniform solute concentrations. All the other tubes exchange with tube 6 as shown in Fig. 1.

This model can be described by the following boundary-value problem. A physiological interpretation of the differential equations is given in [1]. The parameters are chosen according to Farahzad and Tewarson [5].

Differential equations:

$$
\begin{array}{ll}
\frac{d F_{1 v}}{d x}=-J_{1 v}, & J_{1 v}=10\left[\left(c_{61}-c_{11}\right)+\left(c_{62}-c_{12}\right)\right], \\
\frac{d F_{2 v}}{d x}=0, & \\
\frac{d F_{3 v}}{d x}=-J_{3 v}, & J_{3 v}=\left(1-c_{31}\right)+\left(0.05-c_{32}\right), \\
\frac{d F_{4 v}}{d x}=-J_{4 v}, & J_{4 v}=10\left[\left(c_{61}-c_{41}\right)+\left(c_{62}-c_{42}\right)\right],
\end{array}
$$




$$
\begin{aligned}
& \frac{d F_{5 v}}{d x}=0, \\
& \frac{d F_{6 v}}{d x}=J_{1 v}+J_{4 v}, \\
& \frac{d F_{11}}{d x}=0, \\
& \frac{d F_{21}}{d x}=-J_{21}, \quad J_{21}= \begin{cases}1.8, \\
10\left(c_{21}-c_{61}\right),\end{cases} \\
& \frac{d F_{31}}{d x}=-J_{31}, \quad \begin{array}{l}
\gamma \leqslant x \leqslant 1.0 \\
\frac{d F_{41}}{d x}=0,
\end{array} \\
& \frac{d F_{51}}{d x}=-J_{51}, \quad J_{51}=1000\left(c_{51}-c_{61}\right), \\
& \frac{d F_{52}}{d x}=J_{42}+J_{52} .
\end{aligned}
$$

$F_{i k}, F_{i v}$, and $c_{i k}$ are related by the equation

$$
F_{i k}=F_{i v} c_{i k} \quad(i=1, \ldots, 6 ; \quad k=1,2) .
$$


Boundary conditions:

$$
\begin{array}{ll}
F_{1 v}(0)=1, & F_{5 v}(0)=5, \\
c_{11}(0)=1, & c_{51}(0)=1, \\
c_{12}(0)=0.05, & c_{52}(0)=0.05, \\
F_{1 v}(1)=-F_{2 v}(1), & F_{5 v}(1)=-F_{6 v}(1), \\
c_{11}(1)=c_{21}(1), & c_{51}(1)=c_{61}(1), \\
c_{12}(1)=c_{22}(1), & c_{52}(1)=c_{62}(1), \\
F_{2 v}(0)=-F_{3 v}(0), & F_{3 v}(1)=F_{4 v}(0), \\
c_{21}(0)=c_{31}(0), & c_{31}(1)=c_{41}(0), \\
c_{22}(0)=c_{32}(0), & c_{32}(1)=c_{42}(0) .
\end{array}
$$

List of symbols used:

$x \quad$ Independent variable-distance into medulla from corticomedullary border,

$\gamma \quad$ Border between outer and inner medulla,

$k$ Solutes: $k=1$ is salt; $k=2$ is urea,

$F_{i v} \quad$ Axial volume flow in tube $i$,

$F_{i k} \quad$ Axial flow of solute $k$ in tube $i$,

$c_{i k} \quad$ Concentration of solute $k$ in tube $i$,

$J_{i v} \quad$ Outward transmural volume flux from tube $i$,

$J_{i k} \quad$ Outward transmural flux of solute $k$ from tube $i$.

For tube 3 (the DN), $x$ is measured along the tube so that $x=0$ and $x=1$ correspond to its junctions with tube 2 (AHL) and tube 4 (CD), respectively. The flows and concentrations are normalized in that $c_{i k}=1 \simeq 300$ $\mathrm{mOsml}$ and $F_{i v}=1 \simeq 34 \mathrm{nl} / \mathrm{min}$.

The transition from the outer to the inner medulla occurs at the junction of the thin AHL with the thick. Currently, there are two hypothesized mechanisms for urine concentration in the inner medulla, one involving active transport of salt out of the thin AHL ("active model"), the second relying on an entirely passive movement of solutes in the inner zone ("passive model"). In both cases, salt is actively transported across the wall of the thick AHL. The boundary-value problem (2.1) describes a passive model.

An analysis of the ultrastructure of a single loop of Henle in the rabbit kidney demonstrates that a thin AHL makes its transition to its thick part within about 2/1000 of the total medullary thickness (Kaissling and Kriz [6, 
Fig. 28]). Similarly, investigations of the rat kidney show that these transitions occur nearly on the same level (Kriz [7, p. 513]). Therefore, the division between outer and inner medulla is fairly sharp. This coincides with direct measurements of sodium and chloride concentrations in tissue slices of the medulla. Recent electron-probe microanalysis demonstrates a marked change in concentration profiles at the boundary between outer and inner medulla (Lechene and Bonventre [8]). In order to simulate this biological fact in the model, the transmural flux of salt out of tube 2 is assumed to change abruptly at the border $x=\gamma$. This is reflected in a discontinuity in $J_{21}$ and, similarly, in $J_{42}$.

\section{COMPUTATION BY MULTIPLE SHOOTING}

For the numerical solution of the two-point boundary-value problem (2.1) the axial flows $F_{i v}$ and $F_{i k}$ are computed in this paper $(i=1, \ldots, 6$; $k=1,2)$. Thus, the concentrations $c_{i k}$ on the right-hand sides of the differential equations must be replaced by the quotients $F_{i k} / F_{i v}$ according to (2.1.b). The trivial differential equations together with the appropriate boundary conditions immediately yield the following identities:

$$
F_{5 v} \equiv 5, \quad F_{11} \equiv 1, \quad F_{12} \equiv 0.05, \quad F_{22} \equiv-0.05, \quad F_{32} \equiv 0.05 \text {. }
$$

In this manner, the number of differential equations is reduced to 13 . Denoting the vector

$$
\left(F_{1 v}, F_{2 v}, F_{3 v}, F_{4 v}, F_{6 v}, F_{21}, F_{31}, F_{41}, F_{51}, F_{61}, F_{42}, F_{52}, F_{62}\right)^{T}
$$

by $y$, the resulting two-point boundary-value problem may be written in the following compact form $(N=13)$ :

$$
\begin{aligned}
y^{\prime} & =g(x, y), \\
A \cdot b(0)+B \cdot y(1) & =r .
\end{aligned}
$$

Here $g(x, y)$ is vector-valued with components $g_{k}(x, y)(k=1,2, \ldots, N)$, which are functions of the $N+1$ variables $(x, y) . A$ and $B$ are constant square matrices of order $N$, and $r$ is a vector. They are chosen so that (3.1.b) is equivalent to the boundary conditions (2.1.c).

The reduction of the system (2.1) to a system of 13 unknowns makes use of the trivial differential equations. If the number of vanishing right-hand sides in (2.1.a) is reduced (e.g. in order to investigate salt recycling in DHL in the model), the size $N$ of the system (3.1) increases. A simple change of the input parameters of the multiple-shooting routine allows the treatment of such a modification. 
The multiple-shooting method for the solution of a two-point boundaryvalue problem of the form (3.1) is described in [3], [4], and realized in the code [2]. It has been extensively tested in actual computations (see e.g. Diekhoff et al. [9]). Here the interval $0 \leqslant x \leqslant 1$ is suitably subdivided:

$$
0=x_{1}<x_{2}<\cdots<x_{M-1}<x_{M}=1 \quad \text { ( } M \text { nodes). }
$$

Let $y\left(x ; x_{j}, s_{j}\right)(j=1, \ldots, M-1)$ denote the solution of the initial-value problem

$$
y^{\prime}=g(x, y), \quad y\left(x_{j}\right)=s_{j}, \quad x_{j} \leqslant x \leqslant x_{j+1} .
$$

Then the $N$-dimensional vectors $s_{j}$ have to be determined so that the following conditions hold:

continuity conditions (for $M>2$ ):

$$
G_{j}\left(s_{j}, s_{j+1}\right):=y\left(x_{j+1} ; x_{j}, s_{j}\right)-s_{j+1}=0, \quad j=1, \ldots, M-2,
$$

boundary conditions:

$$
G_{M-1}\left(s_{1}, s_{M-1}\right):=A \cdot s_{1}+B \cdot y\left(x_{M} ; x_{M-1}, s_{M-1}\right)-r=0 .
$$

The conditions (3.4) define a system of nonlinear equations

$$
G(s):=\left[\begin{array}{c}
G_{1}\left(s_{1}, s_{2}\right) \\
\vdots \\
G_{M-2}\left(s_{M-2}, s_{M-1}\right) \\
G_{M-1}\left(s_{1}, s_{M-1}\right)
\end{array}\right]=0 \quad \text { with } \quad s:=\left[\begin{array}{c}
s_{1} \\
\vdots \\
s_{M-1}
\end{array}\right] .
$$

This system is solved numerically by the modified Newton method:

$$
\begin{gathered}
s^{k+1}=s^{k}+\lambda_{k} \Delta s^{k}, \quad 0<\lambda_{k} \leqslant 1, \\
\Delta s^{k}=-D G\left(s^{k}\right)^{-1} G\left(s^{k}\right)
\end{gathered}
$$

( $D G(s)$ denotes the Jacobian matrix).

A strategy for choosing the $\lambda_{k}$ has been developed and thoroughly tested by Deuflhard [10]. In order to start the process (3.6) the following initial data must be available:

$$
s_{j}^{0}=\left[\begin{array}{c}
s_{1 j}^{0} \\
\vdots \\
s_{N j}^{0}
\end{array}\right], \quad j=1, \ldots, M-1 .
$$


With the abbreviations

$$
H_{j}:=\frac{\partial y\left(x_{j+1} ; x_{j}, s_{j}\right)}{\partial s_{j}}, \quad j=1, \ldots, M-1,
$$

(3.6.b) can be written in more detail (the $k$-index is omitted):

$$
\left[\begin{array}{ccccc}
H_{1} & -I & & & \\
& H_{2} & -I & 0 & \\
& & \ddots & \ddots & \\
& 0 & & H_{M-2} & -I \\
A & & & & B H_{M-1}
\end{array}\right]\left[\begin{array}{c}
\Delta s_{1} \\
\Delta s_{2} \\
\vdots \\
\Delta s_{M-2} \\
\Delta s_{M-1}
\end{array}\right]=-\left[\begin{array}{c}
G_{1} \\
G_{2} \\
\vdots \\
G_{M-2} \\
G_{M-1}
\end{array}\right] .
$$

The $\Delta s_{j}$ allow a recursive determination by

$$
\begin{aligned}
\Delta s_{1} & =E^{-1} w, \\
\Delta s_{j} & =H_{j-1} \Delta s_{j-1}+G_{j-1}, \quad j=2, \ldots, M-1,
\end{aligned}
$$

with

$$
\begin{aligned}
& E:=A+B H_{M-1} H_{M-2} \cdots H_{1}, \\
& w:=-\left(G_{M-1}+B H_{M-1} G_{M-2}+\cdots+B H_{M-1} \cdots H_{2} G_{1}\right) .
\end{aligned}
$$

At the solution point, the algorithm computes a special norm of the matrix $E$, called norm $(E)$ (cf. [2]). norm $(E)$ represents the sensitivity of the problem relative to the variation of $s_{1}$.

\section{CONSISTENT TREATMENT OF THE DISCONTINUITY}

For the numerical solution of the initial-value problems (3.3), two integration routines were used:

DIFSY1: Bulirsch-Gragg-Stoer extrapolation method (Bulirsch and Stoer [11]) with the stepsize control described in Hussels [12].

RKF7: Runge-Kutta-Fehlberg method of seventh order (Fehlberg [13]).

Both routines control the integration stepsizes automatically according to the prescribed tolerance. ${ }^{1}$ To work well, they require a high order of differentiability on the right-hand side of (3.1.a). $g(x, y)$, however, is of the form [cf. (2.1.a)]

$$
g(x, y)= \begin{cases}g_{1}(x, y), & 0.0 \leqslant x<\gamma, \\ g_{2}(x, y), & \gamma \leqslant x \leqslant 1.0\end{cases}
$$

${ }^{1}$ This control of the stepsizes is based on built-in estimators of the truncation error. 
Because of this discontinuity $x_{k}=\gamma$ was chosen as one of the nodes in (3.2). For the numerical solution of the initial-value problem (3.3) in the interval $x_{k-1} \leqslant x \leqslant x_{k}$, the continuous extension of $g_{1}(x, y)$ to the interval $0 \leqslant x \leqslant \gamma$ has to be used. Then on both sides of $x_{k}=\gamma$ the differential equation is correctly computed. The continuous junction of both parts of the solution curve at $x_{k}=\gamma$ is guaranteed by (3.4.a).

\section{STORAGE REQUIREMENTS}

The storage requirements of the multiple-shooting algorithm are very low. Most of the storage is needed by the $M-1 N \times N$ matrices $H_{j} . M \approx 10$ is sufficient for nearly all purposes. In contrast to finite-difference methods it is not necessary to increase $M$ if higher accuracy is required. In the actual computation of the kidney model $M=12$ and the following nodes in (3.2) were chosen:

$$
\begin{aligned}
& 0.0,0.1,0.2,0.3,0.4,0.45 \text {, } \\
& 0.5,0.6,0.7,0.8,0.9,1.0 \text {. }
\end{aligned}
$$

The tolerance for the integration routines was prescribed as $1_{10}-15$. In this case, the stepsizes selected by the RKF7 algorithm varied between $0.55_{10}-4$ and $0.42_{10}-3$. It seems impossible to achieve the same accuracy by finitedifference methods. Indeed, recalling that RKF7 is of order 7, a finitedifference approximation of order 2 would need a net spacing

$$
h \approx\left(1_{10}-4\right)^{7 / 2}=1 \cdot{ }_{10}-14
$$

in order to yield comparable results. Thus the most economical sparsematrix method (cf. Table 1 in [5]) would require a storage of approximately $\left(2 \times 1_{10} 14\right)^{2}=4_{10} 28$.

\section{COMMENT}

It is shown in [3] that quasilinearization (cf. Bellman and Kalaba [14]) can be obtained from multiple shooting by a limiting process $M \rightarrow \infty$. This method was used by Foster, Jacquez, and Daniels [15] for the computation of a three-tube model of the renal medulla (central-core model).

\section{COMPUTATIONAL RESULTS}

All computations were performed in FORTRAN double precision (96-bit mantissa) on the CDC Cyber 175 of the Leibniz-Rechenzentrum der Bayerischen Akademie der Wissenschaften. The initial data (3.7) were obtained by means of a homotopy method (or continuation method). The use of this technique may be motivated by theoretical investigations (for 
TABLE 1

\begin{tabular}{lrrr}
\hline & \multicolumn{3}{c}{ Results of multiple shooting for $\gamma=$} \\
\cline { 2 - 4 } & \multicolumn{1}{c}{0.4} & \multicolumn{1}{c}{0.45} & \multicolumn{1}{c}{0.5} \\
\hline$F_{10}(1)^{\mathrm{a}}$ & $0.5674811_{10}+00$ & $0.4178138_{10}+00$ & $0.3642430_{10}+00$ \\
$c_{11}(1)$ & $0.1762173_{10}+01$ & $0.2393411_{10}+01$ & $0.2745420_{10}+01$ \\
$c_{12}(1)$ & $0.8810866_{10}-01$ & $0.1196705_{10}+00$ & $0.1372710_{10}+00$ \\
$F_{4 v}(1)^{\mathrm{a}}$ & $0.6176376_{10}-01$ & $0.1509508_{10}-01$ & $0.1280209_{10}-01$ \\
$c_{41}(1)$ & $0.1107612_{10}+01$ & $0.2256045_{10}-01$ & $0.3000758_{10}-03$ \\
$c_{42}(1)$ & $0.7425895_{10}+00$ & $0.2491849_{10}+01$ & $0.2882899_{10}+01$ \\
$F_{6 v}(0)^{\mathrm{a}}$ & $-0.5542662_{10}+01$ & $-0.5615708_{10}+01$ & $-0.5670587_{10}+01$ \\
$c_{61}(0)$ & $0.1025019_{10}+01$ & $0.1044662_{10}+01$ & $0.1049184_{10}+01$ \\
$c_{62}(0)$ & $0.4585070_{10}-01$ & $0.4672346_{10}-01$ & $0.4639606_{10}-01$ \\
\hline
\end{tabular}

asince the axial flows form a countercurrent system, any flow from the corticomedullary border to the papilla is positive, whereas the opposite flow direction must be negative in the model.

details see [16]). The modified Newton iteration (3.6) of the multiple-shooting algorithm was terminated when the solution had achieved a relative precision ${ }^{2}$ of $1_{10}-12$. An important check on the final results is the conservation of mass over the medulla. In these computations the mass inflow agreed with the mass outflow to a relative accuracy of at least $1_{10}-20$ for both water and the two solutes.

As in Sec. 2, $\gamma$ may denote the border between the outer and inner medulla. In Table 1 (truncated) results for various values of $\gamma(\gamma=0.4,0.45$, and 0.5 ) are given. For these values of $\gamma$, norm (E) (cf. Sec. 3) was computed as $3_{10} 13,1_{10} 13$, and $4_{10} 12$. This indicates how extremely sensitive the problem is. Therefore, a careful computation in double precision is necessary in order to get reliable results for this challenging boundary-value problem. Figures 2 and 3 show the computed total solute concentrations in tubes $1-4$ for $\gamma=0.5$.

\section{COMMENT}

In comparison with measured salt concentrations in the final urine, the computed values of $c_{41}(1)$ for $\gamma=0.45$ or 0.5 are too low. $c_{41}(1) \approx 1$ would be closer to reality. The reason for this discrepancy is as follows: In order to concentrate the urine in the inner medulla with the passive mechanism, it was necessary in [1] and [5] to maximize the urea load entering the CD. As

${ }^{2}$ When the algorithm had reached this precision it performed an additional Newton step. So, due to the quadratic convergence, the continuity and boundary conditions are satisfied with an accuracy of about $1_{10}-30$ in these computations. 


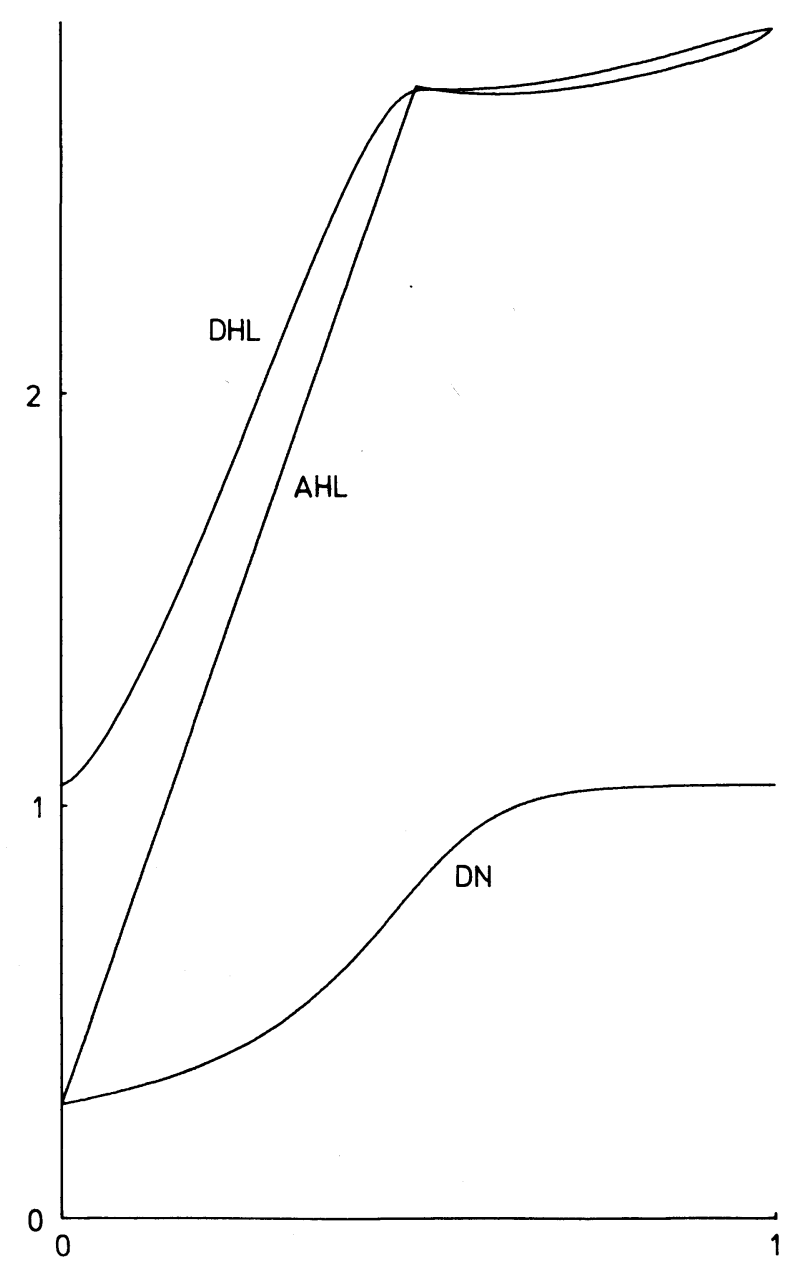

Fig. 2. Total concentrations in DHL $\left(c_{11}+c_{12}\right)$, AHL $\left(c_{21}+c_{22}\right)$, and DN $\left(c_{31}+c_{32}\right)$ for $\gamma=0.5$.

a consequence, the salt concentration at the end of the DN had to be assumed very low to achieve the biologically correct total concentration in the final urine. Therefore, these articles and the present paper use somewhat artificial parameters in the DN.

To compare the results of [1] and [5] with those presented here it is necessary to know that in these papers a somewhat different problem is solved. The computed values of these articles belong to a model with a linear transition zone between the outer and inner medulla such that, for 


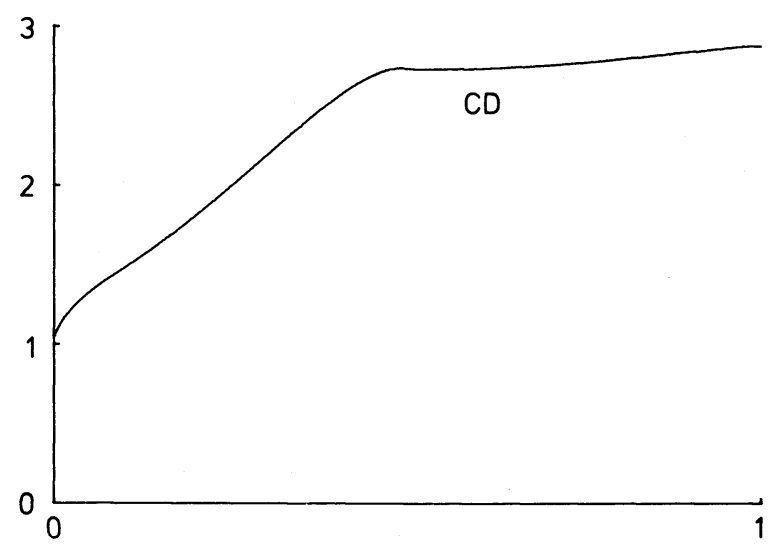

FIG. 3. Total concentration in $\mathrm{CD}\left(c_{41}+c_{42}\right)$ for $\gamma=0.5$.

example,

$$
J_{21}(x)=\left\{\begin{array}{cc}
1.8, & 0.0 \leqslant x<0.4 \\
1.8+m \cdot(x-0.4), & 0.4 \leqslant x \leqslant 0.5 \\
10\left(c_{21}-c_{61}\right), & 0.5<x \leqslant 1.0
\end{array}\right.
$$

where $m=10\left\{10\left[c_{21}(x)-c_{61}(x)\right]-1.8\right\}$. A computation of this problem by the multiple-shooting algorithm yielded the following results:

$$
\begin{aligned}
& F_{1 v}(1)=0.4283387_{10}+00, \\
& c_{11}(1)=0.2334601_{10}+01, \\
& c_{12}(1)=0.1167301_{10}+00, \\
& F_{4 v}(1)=0.1580020_{10}-01, \\
& c_{41}(1)=0.4669535_{10}-01, \\
& c_{42}(1)=0.2406077_{10}+01, \\
& F_{6 v}(0)=-0.5605542_{10}+01, \\
& c_{61}(0)=0.1043765_{10}+01, \\
& c_{62}(0)=0.4673652_{10}-01
\end{aligned}
$$

The author wishes to thank Professor R. Bulirsch, who stimulated and encouraged this work. He is indebted to Professor M. Horster (Physiologisches 
Institut der Universität München) and Dr. L. C. Moore (Health Science Center of the State University of New York) for their valuable help concerning the physiological model.

\section{REFERENCES}

1 J. L. Stephenson, R. P. Tewarson, and R. Mejia, Quantitative analysis of mass and energy balance in non-ideal models of the renal counterflow system, Proc. Nat. Acad. Sci. U. S. A. 71:1618-1622 (1974).

2 R. Bulirsch, J. Stoer, and P. Deuflhard, Numerical solution of nonlinear two-point boundary value problems I, Numer. Math., to appear.

3 J. Stoer and R. Bulirsch, Einführung in die Numerische Mathematik II, Springer, New York, 1978.

4 H. B. Keller, Numerical Methods for Two-Point Boundary-Value Problems, Blaisdell, Waltham, 1968.

5 P. Farahzad and R. P. Tewarson, An efficient numerical method for solving the differential equation of renal counterflow systems, Comput. Biol. Med. 8:57-64 (1978).

6 B. Kaissling and W. Kriz, Structural Analysis of the Rabbit Kidney, Advances in Anatomy, Embryology, and Cell Biology, Vol. 56, Springer, New York, 1979.

7 W. Kriz, Der architektonische und funktionelle Aufbau der Rattenniere, $Z$. Zellforsch. 82:495-535 (1967).

8 C. P. Lechene and J. V. Bonventre, Electron probe study of the urinary concentrating mechanism elemental corticopapillary gradient in frozen hydrated rat kidney, in Proceedings, 11th Annual Conference, Microbeam Analysis Society, 1976, 61 A-61G.

9 H.-J. Diekhoff, P. Lory, H. J. Oberle, H.-J. Pesch, P. Rentrop, and R. Seydel, Comparing routines for the numerical solution of initial value problems of ordinary differential equations in multiple shooting, Numer. Math. 27:449-469 (1977).

10 P. Deuflhard, A relaxation strategy for the modified Newton method, in Optimization and Optimal Control (R. Bulirsch, W. Oettli, and J. Stoer, Eds.), Lecture Notes in Mathematics, Vol. 477, Springer, New York, 1975.

11 R. Bulirsch and J. Stoer, Numerical treatment of ordinary differential equations by extrapolation methods, Numer. Math. 8:1-13 (1966).

12 H.-G. Hussels, Schrittweitensteuerung bei der Integration gewöhnlicher Differentialgleichungen mit Extrapolationsverfahren, Diplomarbeit, Universität zu Köln, Mathematisches Institut, 1973.

13 E. Fehlberg, Klassische Runge-Kutta-Formeln fünfter und siebenter Ordnung mit Schrittweitenkontrolle, Computing 4:93-106 (1969).

14 R. Bellman and R. Kalaba, Quasilinearization and Nonlinear Boundary-Value Problems, American Elsevier, New York, 1965.

15 D. Foster, J. A. Jacquez, and E. Daniels, Solute concentration in the kidney-II. Input-output studies on a central core model, Math. Biosci. 32:337-360 (1976).

16 P. Lory, Homotopieverfahren und Anwendung der Mehrzielmethode auf mathematische Modelle aus der Physiologie, Dissertation, Technische Universität München, Institut für Mathematik, 1978. 